\title{
Surveying the factor structure and reliability of the Persian version of the Jefferson Scale of Physician Lifelong Learning (JeffSPLL) in staff of medical sciences
}

\author{
Fatemeh Zahra Karimi ${ }^{1}$, Aytay Alesheikh ${ }^{2}$, Soheila Pakravan ${ }^{3}$, Mahbubeh Abdollahi $^{4}$, Mozhdeh Damough ${ }^{5}$, \\ Zahra Khosravi Anbaran $^{6}$, Leila Amiri Farahani ${ }^{7}$
}

\footnotetext{
${ }^{1}$ Ph.D., Assistant Professor, Department of Midwifery, Evidence-Based Health Care Research Center, School of Nursing and Midwifery, Mashhad University of Medical Sciences, Mashhad, Iran

${ }^{2}$ M.Sc. of Midwifery, Lecturer, School of Medicine, Islamic Azad University, Branch of Mashhad, Iran

${ }^{3}$ B.Sc. of Midwifery, Mashhad University of Medical Sciences, Mashhad, Iran

${ }^{4}$ Ph.D. in Biostatistics, Torbat Heydariyeh University of Medical Sciences, Torbat Heydariyeh, Iran

${ }^{5}$ B.Sc. of Midwifery, Mashhad University of Medical Sciences, Mashhad, Iran

${ }^{6}$ M.Sc. in Midwifery, School of Nursing and Midwifery, Mashhad University of Medical Sciences, Mashhad, Iran

${ }^{7}$ Ph.D., Assistant Professor, Department of Midwifery, School of Nursing and Midwifery, Iran University of Medical Sciences, Tehran, Iran
}

Type of article: Original

\begin{abstract}
Background: In medical sciences, commitment to lifelong learning has been expressed as an important element. Today, due to the rapid development of medical information and technology, lifelong learning is critical for safe medical care and development in medical research. JeffSPLL is one of the scales for measuring lifelong learning among the staff of medical sciences that has never been used in Iran.

Objectives: The aim of the present study was to determine the factor structure and reliability of the Persian version of JeffSPLL among Persian-speaking staff of universities of medical sciences in Iran.

Methods: This study was a cross-sectional study, methodologically, that was conducted in 2012-2013. In this study, 210 staff members of Birjand University of Medical Sciences were selected. Data collection tool was the Persian version of JeffSPLL. To investigate the factor structure of this tool, confirmatory factor analysis was used and to evaluate the model fit, goodness-of-fit indices, root mean square error of approximation (RMSEA), the ratio of chi-square to the degree of freedom associated with it, comparative fit index (CFI), and root mean square residual (RMR) were used. To investigate the reliability of tool, Cronbach's alpha was employed. Data analysis was conducted using LISREL8.8 and SPSS 20 software.

Results: Confirmatory factor analysis showed that RMSEA was close to 0.1, and CFI and GFI were close to one. Therefore, four-factor model was appropriate. Cronbach's alpha was 0.92 for the whole tool and it was between 0.82 and 0.89 for subscales.

Conclusion: The present study verified the four-factor structure of the 19-item Persian version of JeffSPLL that included professional learning beliefs and motivation, scholarly activities, attention to learning opportunities, and technical skills in information seeking among the staff. In addition, this tool has acceptable reliability. Therefore, it was appropriate to assess lifelong learning in the Persian-speaking staff population.
\end{abstract}

Keywords: Factor Structure; Reliability; JeffSPLL

\section{Introduction}

Learning is an integral part of life and it means to accumulate, think, contemplate, and use complex knowledge, attitudes and skills, such that it enables the person or group to have an active adaptation to the changing environment $(1,2)$. We are living in a rapidly changing world with social, economic, and political complexities that require

\section{Corresponding author:}

Assistant Professor Dr. Leila Amiri Farahani, Department of Midwifery, School of Nursing and Midwifery, Iran University of Medical Sciences, Tehran, Iran.

Tel: +98.2143651808, Fax: +98.2143651820, Email: amirifarahani.1@iums.ac.ir

Received: April 10, 2016, Accepted: September 28, 2017, Published: October 2017

iThenticate screening: August 13, 2016, English editing: October 10, 2017, Quality control: October 12, 2017

(C) 2017 The Authors. This is an open access article under the terms of the Creative Commons Attribution-NonCommercialNoDerivs License, which permits use and distribution in any medium, provided the original work is properly cited, the use is non-commercial and no modifications or adaptations are made. 
adaptation via getting quick insights, skills and new knowledge. An individual will not be able to encounter life challenges unless they become continuous learners, and society will not be sustainable unless it is a learning society (3). Learning is critical to improve the quality of life and lifelong learning assists sustainable development (4). Nowadays, considering rapid changes in technology and global competition paying attention to human resources, lifelong learning is not a novice concept and this idea has existed in many cultures, societies and religions. Lifelong learning is a priority for UNESCO (United Nations Educational, Scientific and Cultural Organization) that has been determined by member countries including Iran to promote the right to education for any person. Lifelong learning for all, as a leading concept and guiding principle, plays an important role in the development and improvement of education (5). Lifelong learning is the acquisition of knowledge, skills, attitudes, and values based on a general desire for perceiving and learning the surrounding concepts, and learning is a complex multi-dimensional social process. Learning, mostly takes place with or through others. Our learning has been included in a developing form throughout our lives, from home, society, and school to the work environment and the wider world (learning leads to a change in our behavior or attitude as an individual or group to the extent we are active). Lifelong learning is the development of human abilities through continuous support that motivates and strengthens people to acquire knowledge, values, and skills and to perceive whatever they need in their lives (6). In medical sciences, commitment to lifelong learning has been expressed as an important element. Today, due to the rapid development of medical information and technologies, lifelong learning is critical for safe medical care and development in medical research and biotechnological development, especially in clinical medicine $(1,7)$. In addition, effective human resource training is one of the main duties of universities. Staff of every university are considered as the precious capital of each nation and considering their academic, research and cultural training, is of significant importance. In addition, proper planning in their education is one of the main duties of universities, since one of the factors of economic, social, and cultural development is comprehensive expansion of the training and education of expert and efficient human resources. The necessity of reviewing the educational programs of the staff of medical universities has been emphasized in response to the rapid changes occurring in the healthcare environment. All staffs of universities of medical sciences, including nurses and physicians should be trained such that they become capable of providing effective care in today's complex healthcare systems. However, despite the emphasis on lifelong learning, there is no general accepted definition. Lifelong learning is a complex and multi-dimensional concept (8, 9). One of the definitions of lifelong learning suggested by the European Lifelong Learning Initiative is as follows: "Lifelong learning is the development of human potentials through a continuous supportive process that prepares people to acquire knowledge, values and skills as well as learning they require through their lives, and stimulates them through confidence, creativity, and pleasure they employ in all roles, conditions, and environments. Another definition for lifelong learning is "a concept that includes a set of self-initiating activities (behavioral) and information seeking skills (ability) that activate a sustainable motivation in people for learning and the ability to know their learning needs (metacognition) $(1,10)$. In this regard, Crowther states that in lifelong learning, the person learns how to learn (11). In the texts related to lifelong learning, Brahmi employs this term as learning how to learn and learning through life (12). From the lifelong learning viewpoint, the staff should be equipped with lifelong learning skills, information literacy, learning how to learn and searching in information resources. In addition, related universities and organizations should provide the grounds to strengthen the learning process and assist the staff to establish learning methods in themselves and acquire scientific and information literacy. In the 21 st century, university officials and planners must work more than ever, since nowadays the major duty of universities is to provide the backgrounds to realize lifelong learning (13). However, despite the importance of lifelong learning, the obstacle currently present in Iran is that there is no tool for evaluating lifelong learning of the staff. Hojat et al. designed the Jefferson Scale of Physician Lifelong Learning (JeffSPLL scale) to measure lifelong learning in physicians of Jefferson Medical College in the U.S (3). Therefore, we decided to validate the Persian version of this scale in the present study. In fact, this study sought to answer the question whether the factor structure and internal consistency of items in JeffSPLL lifelong learning evaluation tool obtained by Hojat et al. is supported for the Iranian staff.

\section{Material and Methods}

\subsection{Research Type and the Study Population}

This is a methodological and validation study conducted in 2013-2014. The statistical population in this study was all staff of medical sciences working at Birjand University of Medical Sciences. Given that, for factor analysis, 10 persons are required for each question in this study, considering the amount of loss, a sample of 210 staffs was considered.

\subsection{Research Tool}

Lifelong learning scale included 19 questions with four choices, and each question was scored between 1 and 4 . Each item was scored in a spectrum of strongly agree (1) to strongly disagree (4). The highest score a subject could 
obtain in this scale was 76 and the lowest score was 19. This tool measures four factors of lifelong learning, including 1- professional learning beliefs and motivation (items $1,2,3,4,5,6,7,8,9$ ) (the highest score $=36$, the lowest score $=9$ ), scholarly activities (items $10,11,12,13$ ) (the highest score $=20$, the lowest score $=5$ ), attention to learning opportunities (items $14,15,16,17$ ) (the highest score $=12$, the lowest score $=3$ ), and technical skills in information seeking (items 18,19$)$ (the highest score $=8$, the lowest score $=2)(1,10)$. The validity and reliability of the Persian version of the lifelong learning scale was approved by Hojat et al. in Jefferson Medical College in the U.S. (3). Since one of the designers of this scale (Hojat MR) was a native Persian speaker, he was asked to send the Persian version of this scale for the authors. In addition, the Persian version was approved using content and formal validity by the research team and several experts and relevant specialists and staff. To determine content and formal validity, the qualitative method was used such that the Persian version of this scale was given to 10 experts associated with the research subject and 10 members of staff who are qualified for the research. Then, their views on simplicity, relevance and clarity, the level of difficulty, inappropriateness and ambiguity were collected based on which required corrections were made.

\subsection{Data Collection}

To collect data, first, subjects were identified and objectives of the study were explained for them. In instance of their consent to participate the study, first, their demographic details were collected and then, JeffSPLL questionnaire was filled by the participants.

\subsection{Research Ethics}

The present study was approved by the Ethics Committee of Birjand University of Medical Sciences. Participants were assured of confidentiality of information and providing the presentation of the results in general and their satisfaction to participate in the study was obtained.

\subsection{Statistical Analysis}

To verify the validity and reliability of the questionnaire, confirmatory factor analysis and Cronbach's alpha were employed respectively. Using LISREL8.8, confirmatory factor analysis was conducted on four-factor model of JeffSPLL, and reliability of the questionnaire was assessed using IBMC SPSS $\odot$ Statistics version 20 (IBMC Corp., Armonk, NY, USA). Maximum likelihood method was used for model estimation and goodness-of-fit indices were used for model fit evaluation. In estimating factor analysis model, the underlying assumptions are, 1- the sample size is sufficient. Since the number of parameters on factor analysis model is always high, a sufficient sample size is required to estimate the parameters of model. In general, the larger the sample size, the better. 2- Data distribution should be normal. 3- Before conducting factor analysis, Kaiser-Meyer-Olkin (KMO) Statistic should be calculated to ensure sampling adequacy. KMO of over 0.8 shows the sampling adequacy. In addition, Bartlett's test of Sphericity was conducted to ensure the justification of factoring. In instance of rejecting the null hypothesis, factoring could be justified $(14,15)$. Confirmatory factor analysis is part of the measuring model that deals with the relations between observed variables and subscales (latent variables) (16). To check the model fit, various indices of fit and structure coefficients of model were used. Fit indices employed, included goodness-of-fit (GFI), root mean square error of approximation (RMSEA), Chi-square and the degree of freedom associated with it, comparative fit index (CFI), and root mean square residual (RMR) that are usually used to determine the fit of confirmatory factor analysis (17). It is worth mentioning that RMSEA index of $\leq 0.06$ shows the goodness-of-fit, between 0.06 and $\leq 0.08$ shows reasonable and acceptable fit, between 0.08 and $>1$ shows medium fit and over 1 shows weak fit of the model. RMR of $\leq 0.08$ shows goodness of fit. GFI and CFI of over 0.9 show good fit. The ratio of Chi-square to the degree of freedom lower than five is acceptable and lower than 3 is good (18). If the factor loading of each item with its structure (subscale) is higher than 0.4 , it is of required accuracy for measurement. If the items of subscales under study have factor loading of less than 0.04 , they are not of necessary significance for measurement and therefore, it is suggested to delete them.

\section{Results}

In this study, 206 staffs of Birjand University of Medical Sciences filled JeffSPLL questionnaire. However, due to the incomplete responses of some of the participants, finally, data related to 171 subjects were statistically analyzed. Mean age of the staff was $35.78 \pm 8.04$. The mean total score of lifelong learning and four subscales of professional learning beliefs and motivation, scholarly activities, attention to learning opportunities, and technical skills in information seeking in the staff, were obtained to be $30.71 \pm 3.89,9.70 \pm 3.94,11.40 \pm 2.65$, and $6.26 \pm 1.46$ respectively. In line with factor structure analysis of JeffSPLL, KMO was calculated to assure the sampling adequacy. In addition, to investigate data correlation matrix being non-zero in the population or in other words, to 
find the justification of factoring, Bartlett's test of sphericity was conducted. Since KMO was over $0.879(0.8)$, and the null hypothesis in Bartlett's test of sphericity with statistic of 1785.841 was rejected at $1 \%$, the sample is adequate and factoring could be justified. Table 1 shows the obtained indices for this model. RMSEA index was close to 0.1 and CFI and GFI indices were close to one. Therefore, the model fit is good. In confirmatory factor analysis, to investigate the validity of questionnaire, maximum likelihood method was used for estimating the model and the following indices were used for model fit: Goodness-of-fit index (GFI), root mean square error of approximation (RMSEA), Chi-square and the degree of freedom associated with it, comparative fit index (CFI), and remained mean root (RMR). In order to investigate about the validity of the four-factor model, a CFA was done. The results of CFA demonstrated that the first model was not good, so it was required to do some corrections on it. The two CFA models are as follows:

1) Model 1: This model was conducted according to the four-factor model of the JeffSPLL scale. As can be seen in Table 1, the indices obtained for this model are $\left(\chi^{2} / \mathrm{df}=3.26, \mathrm{RMR}=0.07\right.$, RMSEA $=0.12$, $\mathrm{GFI}=0.77, \mathrm{CFI}=0.93$ ). These results indicate that the model fit is not good.

2) Model 2: In this model, considering the proposed correction indices, a connection was made between questions 7 and 9 from the first item and questions 10 nd 11 from the second item. As can be seen in Table 1, the indices obtained for this model are $\left(\chi^{2} / \mathrm{df}=2.63\right.$, $\mathrm{RMR}=0.07$, RMSEA $=0.09$, GFI $=0.81$, $\mathrm{CFI}=0.94)$. The obtained indices for the second model show improvement in model fit.

Table 2 shows the standardized factor loadings (beta coefficients). In this table, standardized factor loadings, squared standardized factor loadings (squared multiple correlation coefficient) and t-statistic related to each variable were considered in factor analysis model.

Table 1. A comparison of various models based on goodness-of-fit indices

\begin{tabular}{|l|l|l|l|l|l|l|}
\hline Fit index & $\chi^{2} / \mathrm{df}$ & RMR & RMSEA & GFI & CFI & AIC \\
\hline Model 1 & 3.26 & 0.07 & 0.12 & 0.77 & 0.93 & 565.08 \\
\hline Model 2 & 2.63 & 0.07 & 0.09 & 0.81 & 0.94 & 471.28 \\
\hline
\end{tabular}

Table 2. Standard factor loading, standard error and squared correlation of items in JeffSPLL questionnaire

\begin{tabular}{|c|c|c|c|c|}
\hline Subscale & Question & $\begin{array}{l}\text { Standardized factor } \\
\text { loading }\end{array}$ & $\begin{array}{l}\text { Estimated } \\
\text { standard error }\end{array}$ & $\begin{array}{l}\text { Squared multiple } \\
\text { correlation coefficient }\end{array}$ \\
\hline \multirow{9}{*}{$\begin{array}{l}\text { Professional learning beliefs and } \\
\text { motivation }\end{array}$} & 1 & 0.70 & 0.39 & 0.49 \\
\hline & 2 & 0.80 & 0.36 & 0.64 \\
\hline & 3 & 0.74 & 0.35 & 0.55 \\
\hline & 3 & 0.69 & 0.45 & 0.47 \\
\hline & 5 & 0.65 & 0.40 & 0.43 \\
\hline & 6 & 0.72 & 0.41 & 0.52 \\
\hline & 7 & 0.50 & 0.58 & 0.25 \\
\hline & 8 & 0.70 & 0.44 & 0.48 \\
\hline & 9 & 0.45 & 0.77 & 0.20 \\
\hline \multirow[t]{4}{*}{ Scholarly activities } & 10 & 0.73 & 0.61 & 0.53 \\
\hline & 11 & 0.73 & 0.67 & 0.53 \\
\hline & 12 & 0.89 & 0.41 & 0.80 \\
\hline & 13 & 0.77 & 0.57 & 0.59 \\
\hline \multirow[t]{4}{*}{ Attention to learning opportunities } & 14 & 0.77 & 0.56 & 0.60 \\
\hline & 15 & 0.73 & 0.57 & 0.53 \\
\hline & 16 & 0.68 & 0.60 & 0.46 \\
\hline & 17 & 0.75 & 0.50 & 0.56 \\
\hline \multirow{2}{*}{$\begin{array}{l}\text { Technical skills in information } \\
\text { seeking among the staff }\end{array}$} & 18 & 0.94 & 0.26 & 0.88 \\
\hline & 19 & 0.86 & 0.40 & 0.74 \\
\hline
\end{tabular}

Squared multiple correlation shows a percent of variance of obvious variables that is justified by the factor. A higher factor loading has a higher squared correlation. In each subscale, a higher standard factor loading shows that the related item is a better indicator of that subscale. Results indicate that in all subscales of professional learning beliefs and motivation, scholarly activities, attention to learning opportunities, and technical skills in information seeking, all items have a factor loading of over 0.4. In addition, t-statistic related to numerical items is higher than 1.96. Therefore, there is no need to delete any of the items. However, it appears that in the subscale of professional learning beliefs and motivation, items $1,2,3,6$, and 8 have been better indicators of this subscale. In the subscale of scholarly activities, items 12 and 13 , in the subscale of attention to learning opportunities, items 14,15 and 16 , and 
in the subscale of technical skills in information seeking, item 18 were a better indicator of the related subscale. Therefore, this study verified the four-factor structure of JeffSPLL in the sample of Iranian members of staff. To investigate the reliability of JeffSPLL questionnaire, Cronbach's alpha was used. Cronbach's alpha for the whole questionnaire was 0.92 , for subscale of professional learning beliefs and motivation, it was 0.87 , for scholarly activities, it was 0.87 , for attention to learning opportunities, it was 0.82 and for technical skills in information seeking it was 0.89 .

\section{Discussion}

Findings of this study showed that the subscales extracted from data related to the Persian version of JeffSPLL-MS tool perfectly match the original version. According to the results of confirmatory factor analysis, GFI, RMSEA, and CFI indices are $0.81,0.09$, and 0.94 respectively and this shows the goodness-of-fit. Therefore, it could be concluded that the study by Hojat et al. on providing a tool to measure lifelong learning has been fruitful (3) and this questionnaire is a useful and valid tool that can evaluate lifelong learning. As proposed by Hojat et al., the factor structure of the Persian version of JeffSPLL is also four-factor, and these factors include the subscales of professional learning beliefs and motivation, scholarly activities, attention to learning opportunities, and technical skills in information seeking (3). In the present study, all items have a factor loading of over 0.4 and therefore, none of them were deleted. In the original form, in the subscale of professional learning beliefs and motivation, items 1, 2, 3 , and 4 had the highest factor loading and this is relatively similar to the present study. The difference is that in this study, items 1, 2, 3, 6, and 8 were attributed the highest factor loading. Item 9 had the lowest factor loading in this subscale in this study and in the main research. In the original form, in the subscale of scholarly activities, items 10 , 11, and 12 had the highest factor loadings, while in this study, items 12 and 13 have high factor loadings. In the subscale of attention to learning opportunities in the original form, item 14 was attributed the highest factor loading; while in this study, in addition to item 14, item 17 also had a high factor loading. In addition, in the subscale of technical skills in information seeking in the original form and the present study, item 18 was attributed the highest factor loading. Findings of the study show that the Persian version of JeffSPLL has a fairly good internal consistency. Reliability of the tool in the study by Hojat et al. was obtained with Cronbach's alpha of 0.89 (3); while in this study, the internal consistency was 0.92 for the whole scale. Therefore, since medical education is one of the developing areas, what is most needed is "learning how to learn." As the spirit of lifelong learning, this could make it possible for a person to adapt to the rapid changes of the future. Meanwhile, providing a tool for measuring lifelong learning could be a major step in providing conditions for its establishment. Effective human resource training is considered as one of the main duties of universities. Staff of every university are considered as the precious capital of each nation and considering their academic, research and cultural training, is of significant importance. In addition, proper planning in their education is one of the main duties of the universities, since one of the factors of economic, social, and cultural development is comprehensive expansion of the training and education of expert and efficient human resources. The necessity of reviewing the educational programs of the staff of medical universities has been emphasized in response to the rapid changes occurring in the healthcare environment. All staffs of universities of medical sciences including nurses and physicians should be trained such that they become capable of providing effective care in today's complex healthcare systems. Therefore, to meet the urgent need of medical teams to modern knowledge, those educational methods and techniques should be employed that can promote lifelong learning, the quality of clinical decision-making, and improve the quality of service delivery (19). Therefore, providing the Persian version of JeffSPLL will be effective in achieving the above-mentioned objectives. In connection with research limitations, since the accuracy of subjects in answering questions can be effective on how they respond, to reduce this limitation and to relatively control it, it was attempted to fill the questionnaire at an appropriate time and in a proper and calm environment, and to provide the same conditions in terms of time and environment for the staff to complete the questionnaire. It is of other limitations of this study to be confident about responses provided by subjects. By providing explanations and attracting subjects' attention in this study, it was attempted to ensure that the subjects were honest in answering the questionnaires and reflecting the facts. However, since the questionnaires were filled as self-report by the staff, it is possible that in spite of these measures, the subjects could have hidden some facts, which may have prevented correct response. In addition, since this study is the first research conducted to investigate validity and reliability of the Persian version of JeffSPLL on the staff of Birjand University of Medical Sciences, due to the limitations in terms of generalizability, it is suggested to conduct similar studies in other universities of medical sciences.

\section{Conclusions}

Findings of this study showed that factor structure and reliability of the Persian version of lifelong learning measuring tool was verified in staff of Birjand University of Medical Sciences. The applied importance of this study 
is that due to the lack of a valid and reliable tool according to Iranian culture to measure lifelong learning, the tool investigated in this study appears to be appropriate. It is suggested to conduct more research on its application in other universities of medical sciences in Iran.

\section{Acknowledgments:}

Hereby, Birjand University of Medical Sciences, and Mohammad Reza Hojat MD, the reverend professor of Jefferson Medical College are appreciated for their cooperation and valuable guidance in preparing the research tool in this study.

\section{Conflict of Interest:}

There is no conflict of interest to be declared.

\section{Authors' contributions:}

All authors contributed to this project and article equally. All authors read and approved the final manuscript.

\section{References:}

1) Iranian National Commission for UNESCO. Tehran Cluster Office; 2013. Available from:

2) Bayat S, Gholi poor A, Poor Ezzat A. Factors Affecting the Incidence of Learning City in the 6th District of Tehran. Urban management. 2013; 11(31): 139-56.

3) Hojat M, Veloski J, Nasca TJ, Erdmann JB, Gonnella JS. Assessing Physicians' Orientation Toward Lifelong Learning. J Gen Intern Med. 2006; 21(9): 931-6. doi: 10.1111/j.1525-1497.2006.00500.x. PMID: 16918737, PMID: PMC1831612.

4) Gonnella JS, Callahan CA, Louis DZ, Hojat M, Erdmann JB. Medical education and health services research: the linkage. Med Teach. 2004; 26(1): 7-11. doi: 10.1080/0142159032000156515. PMID: 14744686.

5) Mohebhosseini M, Pirouznik M, Pourhadi P. Workshop on Lifelong Learning in the IR of Iran: Challenges, Achievements and Future Prospect. Education Department Iranian Commission for UNESCO. 2013.

6) Bayat S, Gholipour A, Pourezat A. Factors Affecting the Incidence of Learning City in the 6th District of Tehran. Urban Management. 2013; 11(31): 139-59.

7) Hojat M, Veloski JJ, Gonnella JS. Measurement and Correlates of Physicians'Lifelong Learning. Acad Med. 2009; 84(8): 1066-74. doi: 10.1097/ACM.0b013e3181acf25f. PMID: 19638773.

8) McKenzie P. How to make lifelong learning a reality: implications for the planning of educational provision in Austria. In: Aspin D, Chapman J, Hatton M, Sawano Y, eds. International Handbook of Lifelong Learning. Dordecht, the Netherlands: Kluwer; 2001: 367-78. doi: 10.1007/978-94-010-0916-4-20

9) Longworth N. Learning communities for a learning century. In: Aspin D, Chapman J, Hatton M, Sawano Y, eds. International Handbook of Lifelong Learning. Dordecht, the Netherlands: Kluwer. 2001: 591-618. doi: 10.1007/978-94-010-0916-4-30.

10) Hojat M, Veloski JJ, Gonnella JS. Physician Lifelong Learning: Conceptualization, measurement and correlates in full-time clinicians and academic clinicians. Handbook of Lifelong Learning Developments. Nova Science Publishers. 2009.

11) Afzalnia MR, Oshkooh H, Kalavi A. [Evaluation of Formal, Informal and Lifelong Learning Role in the Age of Instable Information]. Iranian Journal of Engineering Education. 2010; 12(45): 57-63.

12) Mohammadi Mehr M, Maleki H, Abbas Pour A, Khoshdel AR. Role of higher education in lifelong learning. Bimonthly Educational Strategies in medical science. 2011; 4(2): 91 -4.

13) Karimi S, Nasr Esfahani A, Boghratian K. [Manabe va khadamate elmi pajouheshie mowrede niaze daneshgah baraye tahaghoghe yadgiriye madamolomr]. Faslnameye ketab. 2005; (65): 163-184.

14) Sheskin D. Handbook of Parametric and Nonparametric Statistical Procedures. Western Connecticut State University. Chapman \& Hal VCRC. 2004.

15) Sharma S. Applied Multivariate Techniques. New York Chichester Brisbane Toronto Singapore: John Wiley \& Sons. 1996.

16) Aroijan KJ, Norris AE. Confirmatory Factor Analysis. In: Munro BH. Statistical Methods for Health Care Research. 5th ed. New York: Lippincott. 2005; 351-75.

17) Tabachnick BG, Fidell LS. Using multivariate statistics. 5th ed. Boston: Allyn and Bacon. 2007.

18) Byrne BM. Structural equation modeling with LISREL, PRELIS, and SIMPLIS: Basic concepts, applications, and programming. Mahwah, NJ: Lawrence Erlbaum Associates. 2006.

19) Soltani Arabshahi SK, Naeimi L. [Assessment of the current situation of - self-directed learning skills in medical students]. Razi Journal of Medical Sciences. 2013; 20(113): 10-9. 